Psychotherapeut 2011 · 56:6-7

DOI 10.1007/s00278-010-0798-8

Online publiziert: 12. Dezember 2010

(c) Springer-Verlag 2010

\section{Wolfgang Schneider}

Klinik und Poliklinik für Psychosomatik und Psychotherapeutische

Medizin, Zentrum für Nervenheilkunde der Universität Rostock

\title{
Psychische Gesundheit und Arbeit
}

Die öffentliche Aufmerksamkeit für die Relevanz psychischer und psychosomatischer Erkrankungen ist im letzten Jahrzehnt deutlich gewachsen. Epidemiologische Schätzungen der World Health Organization (WHO) gehen davon aus, dass die Jahresprävalenz psychischer und psychosomatischer Erkrankungen der europäischen Bevölkerung bei ca. 30\% liegt, und Vertreter der WHO betonen, dass die Depressionen heute zu einer der drei wichtigsten Volkskrankheiten gehören. Wir lesen in der Presse immer wieder, dass die Arbeitsunfähigkeits- (AU-)Tage wegen psychischer Erkrankungen massiv gegenüber den AU-Tagen wegen anderer Erkrankungen zugenommen haben oder dass sich die Verschreibungen von Antidepressiva in den letzten 10 Jahren (Techniker Krankenkasse 2010) verdoppelt haben. Die besondere volkswirtschaftliche Bedeutung resultiert nicht nur aus den Kosten, die für die medizinische und rehabilitative Versorgung der Menschen mit psychischen und psychosomatischen Erkrankungen anfallen, sondern auch aus den Produktivitätsausfällen oder -einbußen, die sich durch krankgeschriebene Arbeitnehmer (Absentismus), aber auch durch Arbeitnehmer, die zwar am Arbeitsplatz anwesend sind, jedoch aufgrund ihrer psychischen Befindlichkeit in ihrer arbeitsbezogenen Leistung und Kreativität nachhaltig eingeschränkt sind, ergeben.

Die psychischen und psychosomatischen Erkrankungen stellen mittlerweile seit einer Reihe von Jahren den größten Anteil bei den Renten wegen verminderter Erwerbsfähigkeit dar. Führend sind dabei die Depressionen, jedoch sind auch Sucht- und Angsterkrankungen sowie Körperbeschwerden ohne eine organisch zu fassende Grundlage zahlenmäßig bedeutsam. Da auch bei chronischen Erkrankungen den psychosozialen Faktoren eine verlaufsrelevante Funktion zukommt, resultieren über die Rentenzahlungen allein immense volkswirtschaftliche Kosten.

Arbeitslosigkeit stellt ein hohes Risiko für die psychosoziale Gesundheit der Betroffenen dar; hierbei zeigen empirische Befunde, dass v. a. Langzeitarbeitslose (länger als 2 Jahre) vermehrt unter psychischen und psychosomatischen Erkrankungen leiden, aber auch eine höhere Morbidität in Bezug auf körperliche Krankheiten aufweisen. Auch wenn die Bundesregierung zurzeit stolz darauf verweist, dass die Zahl der Arbeitslosen bedeutend zurückgegangen sei, bleibt doch festzustellen, dass sich ein großer Teil der in das Erwerbsleben passager zurückgekehrten Arbeitnehmer in prekären Arbeitskonstellationen (finanziell und inhaltlich) befindet, die sicherlich keine gute Voraussetzung für eine befriedigende psychosoziale Befindlichkeit bieten. Darüber hinaus ist die Zahl der Langzeitarbeitslosen - und hiervon betroffen sind v. a. auch ältere Arbeitnehmer - stabil geblieben. Von Interesse sind Befunde, die darauf hinweisen, dass die psychische Gesundheit von erwerbstätigen Individuen deutlich besser ist als bei den Arbeitslosen (Moser u. Pauli 2009). Wobei die hier zitierten Autoren auf der Grundlage einer Metaanalyse von europäischen Studien zur gesundheitlichen Situation von Ar- beitslosen davon ausgehen, dass der Langzeitarbeitslosigkeit auch eine kausale Wirkung bei der Entstehung von psychischen und psychosomatischen Erkrankungen zukommt.

Die heute oftmals formulierte These, dass psychische Erkrankungen an Häufigkeit zunehmen würden, kann jedoch auch durch die erhöhte öffentliche Sensibilität für die Wahrnehmung und Benennung von psychosozialen Problemen sowie die erhöhte Kompetenz und Bereitschaft von Ärzten zu einer entsprechenden Diagnosestellung mitbeeinflusst sein. Dazu kommt sicherlich, dass sich in den letzten Jahrzehnten die Menschen offener für Gefühle und innerpsychische Prozesse zeigen und wohl bereiter sind als früher, entsprechende Probleme zu „outen“.

Dabei wird gerade den Arbeitsbedingungen in einer globalisierten Welt, die nur noch nach dem Interesse des unkontrollierbaren Finanzkapitals beherrscht werden würde, eine besonders destruktive Wirkung auf die psychische Befindlichkeit der Menschen zugeschrieben. Tatsächlich haben die Veränderungen in der Arbeitswelt in den letzten Jahrzehnten zunehmend zu einem Ansteigen psychischer und sozialer Belastungen und Herausforderungen geführt, sodass diese in vielen Sektoren der Arbeitswelt, die klassischen physikalisch-chemischen oder mechanischen Belastungen und Risiken der industriellen Arbeit in ihrer Bedeutsamkeit für die Gesundheit zurückgedrängt haben. Arbeitsprozesse haben sich verdichtet, und durch die elektronische Kommunikation in einer Wissenschaftsgesellschaft sind sie schneller und komplexer gewor- 
den. Die vielfältigen Aufgaben im Dienstleistungssektor (Bildung, Krankenhäuser, Pflegeeinrichtungen etc.) erfordern viel in Bezug auf die emotionalen und interaktionellen Kompetenzen der Arbeitnehmer. Gefordert sind unter den Arbeitsbedingungen der postmodernen Gesellschaft vom Individuum die Bereitschaft und Fähigkeit zu beruflichen Veränderungen und lebenslanger Weiterbildung. Der Arbeitnehmer muss ein hohes Ausmaß an inhaltlicher und räumlicher Mobilität und Flexibilität aufbringen, neue Aufgaben und Tätigkeiten übernehmen sowie Orte und soziale Kontexte aufgeben und sich neue soziale Netze schaffen.

Für die Individuen hat sich mittlerweile vor dem Hintergrund der skizzierten Entwicklungen oftmals eine unsichere Arbeitsbiografie herausgebildet, die auch zu einer Verunsicherung der sozialen Identität führen kann. Die Bewältigung dieser Herausforderungen gelingt häufig nicht und führt zu individuellen Beeinträchtigungen, aber ebenso zum Zusammenbrechen von sozialen Beziehungen, wie die hohen Scheidungsziffern zeigen. Der gesellschaftliche Bezugsrahmen bietet so insgesamt wenig Struktur, und die praktische Bedeutung von wertestabilisierenden Institutionen hat abgenommen. Dies erfordert von den Individuen ein hohes Ausmaß an innerer Regulationsfähigkeit und Struktur, um eine angemessene Selbststeuerung in Bezug auf die Realisierung individueller Bedürfnisse und einer - sozial angemessenen - Integration in die Gesellschaft zu erlangen. Es hat den Anschein, dass viele Menschen an der Bewältigung der psychosozialen Herausforderungen der Gesellschaft scheitern (Ehrenberg 2004). So nimmt der Begriff des „burn-out“ mittlerweile in den öffentlichen Medien einen breiten Raum ein, und viele Unternehmen und Organisationen sind für entsprechende Themen sensibilisiert und suchen Unterstützung bei psychosozialen Experten, um die Produktivitäts- und Kreativitätseinbußen ihrer Führungskräfte zu minimieren. Gefragt sind dann entsprechende „Coaching“-Angebote, Stressbewältigungsprogramme oder Workshops zur „work-life balance“.

Aber auch für Psychotherapeuten stellen die Probleme, die sich für die Indi- viduen aus ihren aktuellen Lebens- und Arbeitsbedingungen ergeben, eine große Herausforderung dar, mit der sie oftmals nicht gelernt haben, angemessen umzugehen. Psychotherapie orientiert sich oftmals zu sehr an den traditionellen Krankheitskonzepten, die insgesamt zu wenig Bezug nehmen auf aktuelle und chronische Belastungen im „Hier und Jetzt“, die sich aus den besonderen Arbeits- und Lebensbedingungen von Individuen ergeben. Dabei werden lebensgeschichtlich erworbene konflikthafte Themen, persönlichkeitsstrukturelle Defizite oder dysfunktionale Lernprozesse fokussiert und in der Therapie zu bearbeiten versucht und die aktuell wirksamen dysfunktionalen und hilflos oder perspektivlos machenden Lebensverhältnisse und Ansätze ihrer Bewältigung vernachlässigt. In Diskussionen zum Thema "Arbeit und psychische Gesundheit“ haben mir wiederholt Psychotherapeuten berichtet, dass die sozialen Problemlagen ihrer Patienten die psychotherapeutische Arbeit in relevanter Weise beeinflussen bzw. es notwendig machen, diese in der Behandlung aufzugreifen und zu bearbeiten. Nur fehlen allzu oft angemessene Konzepte und Handlungsansätze, die Dialektik des Ineinanderwirkens von frühen psychosozialen Einflussfaktoren und Wirkvariablen, die sich aus der aktuellen psychosozialen Lebenswelt ergeben, zu verstehen und dieser effektive Handlungsansätze entgegenzusetzen. Es muss wohl auch eingeräumt werden, dass oftmals Psychotherapie allein nicht hinreicht, die psychosozialen „Notlagen“ von Individuen, die sich z. B. in psychischen und psychosomatischen Erkrankungen äußern, zu beheben. Es geht zunehmend darum, die soziale Dimension im Behandlungsprozess zu reflektieren und aufzugreifen. Dafür ist eine Vernetzung mit anderen „psychosozialen“ Akteuren (z. B. Betriebsärzten) notwendig, um möglichst eine positive Beeinflussung der psychosozialen Situation der Patienten zu erreichen.

\section{Korrespondenzadresse}

Prof. Dr. Dr. Wolfgang Schneider Klinik und Poliklinik für Psychosomatik und Psychotherapeutische Medizin, Zentrum für Nervenheilkunde der Universität Rostock Gehlsheimer Str. 20, 18147 Rostock wolfgang.Schneider@med.uni-rostock.de
Schwerpunktthemen

Das Herausgebergremium der Zeitschrift Psychotherapeut lädt Autorinnen und Autoren ein, an den geplanten Schwerpunkten mitzuarbeiten und geeignete Manuskripte einzureichen. Diese werden dem üblichen Reviewverfahren unterzogen. Bitte schicken Sie Ihren Beitrag an die Redaktion:

Regine.Karcher-Reiners@springer.com

Die Schwerpunktplanung ist vorläufig und kann kurzfristig umdisponiert werden.

Heft 4/11: Gruppenpsychotherapie (Deadline 18.3.2011)

Heft 5/11: Psychoonkologie (Deadline 25.5.2011)

Heft 6/11: Psychotherapie und elektronische Medien

(Deadline 26.7.2011)

Heft 1/12: Migration

(Deadline: 17.9.2011)

Heft 2/12: Themata der Lindauer Psychotherapiewochen 2011

(Deadline 18.11.2011)

Heft 3/12: Neurowissenschaften (Deadline 14.1.2012) 